\title{
The energetically optimal cadence decreases after prolonged cycling exercise
}

\section{Other Journal Item}

\section{Author(s):}

Annaheim, Simon; Boutellier, Urs; Kohler, Goetz

Publication date:

2010-08

Permanent link:

https://doi.org/10.3929/ethz-b-000156493

\section{Rights / license:}

In Copyright - Non-Commercial Use Permitted

Originally published in:

European Journal of Applied Physiology 109(6), https://doi.org/10.1007/s00421-010-1431-9 


\title{
The energetically optimal cadence decreases after prolonged cycling exercise
}

\author{
Simon Annaheim • Urs Boutellier • Götz Kohler
}

Accepted: 5 March 2010/Published online: 6 April 2010

(C) Springer-Verlag 2010

\begin{abstract}
This study investigated the change in the energetically optimal cadence after prolonged cycling. The energetically optimal cadence (EOC) was determined in 14 experienced cyclists by pulmonary gas exchange at six different cadences (100-50 rpm at $10 \mathrm{rpm}$ intervals). The determination of the EOC was repeated after a prolonged cycling exercise of $55 \mathrm{~min}$ duration, where cadence was fixed either at high $(>95 \mathrm{rpm})$ or low $(<55 \mathrm{rpm})$ pedalling rates. The EOC decreased after prolonged cycling exercise at a high as well as at a low fixed cadence $(P<0.01)$. According to the generalized muscle equations of Hill, this indicates that most likely more type I muscle fibres contribute to muscular power output after fatiguing cycling exercise compared to cycling in the beginning of an exercise bout. We suggest that the determination of EOC might be a potential non-invasive method to detect the qualitative
\end{abstract}

Communicated by Susan Ward.

S. Annaheim $(\bowtie) \cdot$ U. Boutellier

Exercise Physiology, Institute of Human Movement Sciences, ETH Zurich, Winterthurerstrasse 190, 8057 Zurich, Switzerland e-mail: simon.annaheim@physiol.biol.ethz.ch

U. Boutellier

Institute of Physiology, Zurich Center for Integrative Human Physiology (ZIHP), University of Zurich, Winterthurerstrasse 190, 8057 Zurich, Switzerland

\section{G. Kohler}

Radiation Oncology, University Hospital of Basel, 4031 Basel, Switzerland

G. Kohler

Biophysical Chemistry, Biocenter, University of Basel, Klingelbergstrasse 70, 4056 Basel, Switzerland changes in activated muscle fibres, which needs further investigation.

Keywords Cycling $\cdot$ Pedalling rate $\cdot$ Endurance exercise . Muscle fibre type

\section{Introduction}

Cyclists chose their pedalling rate freely, independent of cycling speed or power output. Besides the freely chosen cadence, the energetically optimal cadence (EOC), which minimises energy expenditure at a given workload, is of great interest in sport science. Several investigations found that EOC is not fixed but increases with increasing workload (Coast and Welch 1985; di Prampero 2000; Foss and Hallén 2004).

The generalised Hill equations explain why EOC increases with greater workloads (Kohler and Boutellier 2005). These fundamental relationships show that for any muscle consisting of both types of muscle fibres, the most efficient muscle shortening velocity depends on muscle fibre composition. The most efficient muscle shortening velocity in muscles containing many type I muscle fibres is slower compared to muscles of the same length consisting of many type II muscle fibres (Hill 1938, 1964).

Since Martin et al. (2000) showed that muscle function during cycling is similar to that observed in situ, cadence can be used for equivalent muscle shortening velocity in Hills' relationships. With increasing workload, the force production of locomotor muscles has to increase, which results in the additional activation of type II muscle fibres (Henneman et al. 1965). Therefore, we suggest that activated muscle fibres contributing to muscular force production are a major factor affecting EOC. 
However, workload itself is not the only parameter that leads to modulations in activation of muscle fibres. Endurance cycling may act as another external demand, which induces changes in activated muscle fibres. Muscle fibre types differ due to their fatiguability. Thus, we expect alterations in activated muscle fibres as endurance exercise proceeds.

The aim of this study was to investigate the change in EOC after prolonged cycling. We hypothesise that the pattern of activated muscle fibres changes such that fatigue-resistant type I muscle fibres are activated predominantly after prolonged cycling. This will reduce EOC according to the generalised Hill relationships.

\section{Methods}

\section{Subjects}

Fourteen healthy experienced cyclists participated in the study after giving their written informed consent. Cardiovascular and pulmonary diseases as well as medication, detected by a questionnaire, were exclusion criteria.

The subjects' anthropometric and physiological characteristics were as follows (values are mean \pm standard deviation): age $31.5 \pm 8.4$ years; height $179.5 \pm 6.7 \mathrm{~cm}$; body mass $73.1 \pm 8.0 \mathrm{~kg} ; \dot{V} \mathrm{O}_{2 \max }: 58.1 \pm 8.7 \mathrm{ml} \mathrm{kg}^{-1}$ $\min ^{-1}$; maximal power reached during incremental test $4.9 \pm 0.9 \mathrm{~W} \mathrm{~kg}^{-1}$; workload at ventilatory threshold $4.0 \pm 0.7 \mathrm{~W} \mathrm{~kg}^{-1}$.

The experimental design was approved by the Human Ethics Committee of the ETH Zurich.

\section{Experimental design}

The investigations were conducted over three sessions. In the first session, maximal oxygen uptake $\left(\dot{V} \mathrm{O}_{2 \max }\right)$ was determined and a familiarisation trial of the EOC test protocol was conducted. After a recovery period of at least $48 \mathrm{~h}$, EOC and its change after prolonged cycling was determined during sessions 2 and 3 (Fig. 1), which were separated by at least 7 days.

Subjects were asked to refrain from intensive exercise for at least $24 \mathrm{~h}$ before each session. The sessions took place in the morning after the subject had eaten a carbohydrate-rich breakfast. No energy intake was allowed during the test sessions, since carbohydrate availability may alter the pattern of activated muscle fibres. Water intake was permitted ad libitum. A similar regimen of longer duration has already been applied in a previous study, and no cases of hypoglycaemia (blood glucose concentration $<50 \mathrm{mg} / \mathrm{dl}$ ) were detected (Kohler and Boutellier 2004). All subjects of this study tolerated the protocol well without any occurrence of hypoglycaemia.

\section{Exercise protocols}

The cycling exercises were conducted on an electronically braked bicycle ergometer (ergoselect 200k, Ergoline, Bitz, Germany), which allowed power output adjustment independently of cadence. An incremental cycling test protocol was used to determine $\dot{V} \mathrm{O}_{2 \max }$. Initial power output was set at $100 \mathrm{~W}$ and increased by $30 \mathrm{~W}$ every $2 \mathrm{~min}$ until exhaustion. Cadence was kept constant at $80 \mathrm{rpm}$ throughout the test. Respiratory data were recorded breath by breath (Oxycon beta, Jaeger, Hoechberg, Germany) and sampled as mean values over $10 \mathrm{~s}$. Mean oxygen consumption over the last $30 \mathrm{~s}$ before exhaustion was taken as $\dot{V} \mathrm{O}_{2 \max }$. Ventilatory threshold was determined based on ventilatory (minute ventilation) and respiratory data (respiratory exchange ratio).

The determination of EOC was performed at a workload corresponding to $65 \% \quad \dot{V} \mathrm{O}_{2 \max }(210 \pm 35 \mathrm{~W})$ and was carried out after a warm-up of $15 \mathrm{~min}$ at a workload

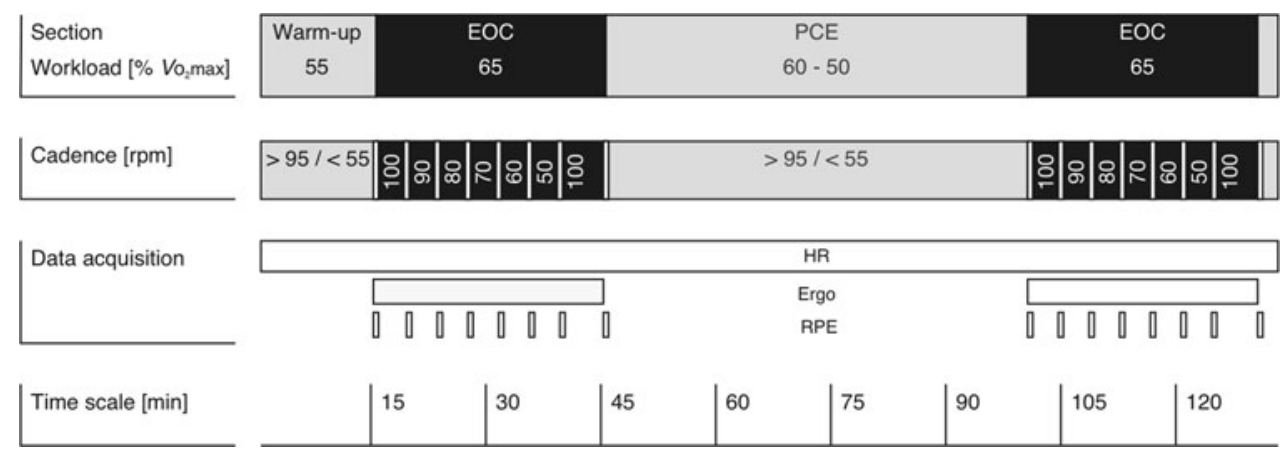

Fig. 1 Overview of experimental cycling protocol applied at test sessions 3 and 4: workload, cadence and data acquisition during warm-up, at the determination of energetically optimal cadence
(EOC) and at prolonged cycling exercise (PCE). The second determination of EOC was followed by a cool down of $2 \mathrm{~min}$. $H R$ heart rate, Ergo ergospirometry, $R P E$ rate of perceived exertion 
corresponding to $55 \% \quad \dot{V} \mathrm{O}_{2 \max }$. For each subject, the workload was below the ventilatory threshold. Respiratory data were acquired at six different cadences: starting at $100 \mathrm{rpm}\left(100 \mathrm{rpm}_{\text {start }}\right)$, cadence was reduced every $4 \mathrm{~min}$ by $10 \mathrm{rpm}$. After completing $50 \mathrm{rpm}$, cadence was increased again up to $100 \mathrm{rpm}$ for a further $6 \mathrm{~min}(100$ $\left.\mathrm{rpm}_{\text {end }}\right)$. The given cadences had to be maintained as closely as possible and were monitored continuously to verify accuracy. Heart rate was recorded continuously (Polar s610i; Polar Electro, Kempele, Finland). Additionally, subjects were asked to rate their perceived exertion according to a modified Borg scale containing a numerical range from 0 to 10 (Borg 1982).

A prolonged cycling exercise followed the determination of EOC during sessions 2 and 3 and was conducted at either a high (above $95 \mathrm{rpm}$ ) or a low cadence (below $55 \mathrm{rpm}$ ), because the cadence during the prolonged cycling exercise might affect EOC by conditioning. The order of cadences set during the prolonged cycling exercise at sessions 2 and 3 was randomised. The prolonged cycling exercise started at a workload corresponding to $60 \%$ $\dot{V} \mathrm{O}_{2 \max }$. However, the initial workload had to be lowered to $55 \% \quad \dot{V} \mathrm{O}_{2 \max }$ as prolonged cycling exercise progressed to ensure the completion of the whole session. The reduction in workload occurred after $49.9 \pm 3.9$ and $47.8 \pm 7.5 \mathrm{~min}$ during prolonged cycling exercise at a high and low cadence, respectively. The criterion for the workload reduction was either an increase in heart rate above $85 \%$ of maximal heart rate attained during the incremental cycling test or the feeling of the subjects that they might not be able to complete the remaining exercise protocol, i.e. the determination of EOC.

After the prolonged cycling exercise, EOC was determined again, which summed to a total cycling exercise time of 132 min during sessions 2 and 3.

Data processing and statistics

Energy expenditure (EE) during the determination of EOC was calculated from oxygen uptake and carbon dioxide exhalation by indirect calorimetry according to the table of thermal equivalents of oxygen for the non-protein respiratory exchange ratio (Zuntz 1901). Only data of the last 2 min of each cadence step were included for calculation, assuring respiratory steady state. Values of EE were plotted against its corresponding cadence and approximated by a parabola for each subject. This form has previously been used in several studies and seems to best fit this relationship (Brisswalter et al. 2000; Chavarren and Calbet 1999; Hansen et al. 2002). Degree of fit $\left(R^{2}\right)$ ranged from 0.68 to 1.00 with the median at $0.97(n=56)$. Minimal EE and concomitantly EOC were calculated analytically from the parabolic curve equation. Against our expectations, some subjects showed EOC values below the range of measurement (50-100 rpm). To ensure an accurate analysis of the shift in EOC after prolonged cycling exercise, we set the criterion that EOC values determined in one session had to lie inside the range of measurement (between 50 and $100 \mathrm{rpm}$ ) at least once. Otherwise, EOC values determined in this session were excluded from statistical analysis. Following this criterion, EOC values from 4 out of 28 (14\%) sessions had to be excluded.

Data were checked for normality of distribution (Kolmogorov-Smirnov test) and homogeneity of variance (Levene's test for equality of variances). Data samples of EOC for each subject did not meet the guidelines. Thus, a binomial test was conducted to make a qualitative statement about the direction of the EOC shift after prolonged cycling exercise. The other physiological parameters acquired during the determination of EOC were tested for differences by MANOVA for repeated measures. Statistical analysis was performed using SPSS 13.0 for Mac OS X (Chicago, Illinois, USA). Level of significance was set at $P<0.05$. Data are presented as mean \pm standard deviation.

\section{Results}

Energetically optimal cadence

Mean EE of all subjects $(n=14)$ during determination of EOC before and after the prolonged cycling exercise is shown in Fig. 2. The minima of the approximated parabola, representing EOC, were found at 59.2 and $47.9 \mathrm{rpm}$ with prolonged cycling exercise at high cadence as well as 58.9 and $37.4 \mathrm{rpm}$ at low cadence.

Analysing the change in EOC separately in each subject, EOC was reduced in 11 out of 13 and in 9 out of 11 subjects after prolonged cycling exercise at a high and a low cadence, respectively. Finally, this led to a statistically significant decrease in cadence $(P<0.01)$ after the prolonged cycling exercise.

\section{Oxygen consumption and energy expenditure}

Energy expenditure (Fig. 2) was unchanged after the prolonged cycling exercise. Oxygen consumption increased significantly from $100 \mathrm{rpm}_{\text {start }}$ to $100 \mathrm{rpm}_{\text {end }}$ before the prolonged cycling exercise, whilst this increase was less pronounced after the prolonged cycling exercise (Table 1).

\section{Energy metabolism}

Respiratory exchange ratio (RER) decreased significantly after the prolonged cycling exercise at a high as well as at a low cadence (Fig. 3). 
Fig. 2 Energy expenditure during determination of energetically optimal cadence (EOC) before (white diamonds) and after (black diamonds) prolonged cycling exercise (PCE), which was performed either at high $(>95 \mathrm{rpm}, \mathbf{a})$ or low cadence $(<55 \mathrm{rpm}, \mathbf{b})$. Data are presented as mean and standard deviation $(n=14)$. There was no statistically significant change in energy expenditure after PCE at high $(P=0.536)$ as well as at low cadence $(P=0.054)$. White arrow EOC before PCE; Black arrow EOC after PCE

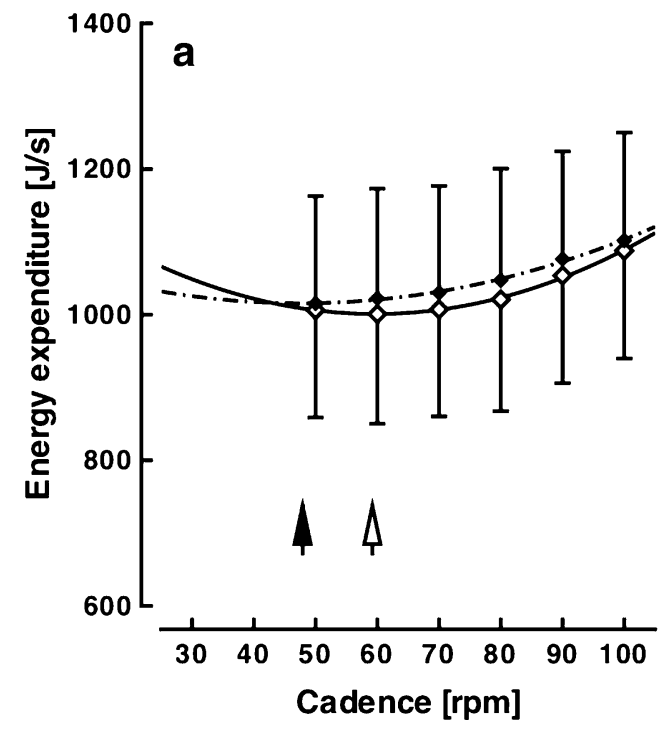

b
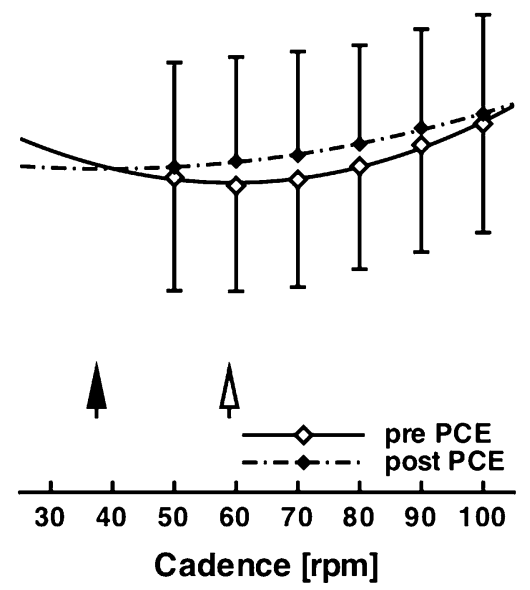

Table 1 As depicted in Fig. 1, Oxygen consumption $\left(\dot{V} \mathrm{O}_{2}\right)$ and minute ventilation $(\dot{V} \mathrm{E})$ at 100 rpm were measured twice during determination of energetically optimal cadence (EOC), whilst EOC was determined prior to (Pre-PCE) and after prolonged cycling exercise (Post-PCE)

\begin{tabular}{|c|c|c|c|c|c|c|}
\hline & \multicolumn{3}{|l|}{ Pre-PCE } & \multicolumn{3}{|l|}{ Post-PCE } \\
\hline & $100 \mathrm{rpm}_{\text {start }}$ & $100 \mathrm{rpm}_{\text {end }}$ & $\Delta$ & $100 \mathrm{rpm}_{\text {start }}$ & $100 \mathrm{rpm}_{\text {end }}$ & $\Delta$ \\
\hline \multicolumn{7}{|c|}{$\dot{V} \mathrm{O}_{2}\left(\mathrm{ml} \cdot \min ^{-1}\right)$} \\
\hline $\mathrm{PCE}_{\text {high }}$ & $3140 \pm 409$ & $3247 \pm 413$ & $+3.5 \pm 2.7 \% * *$ & $3226 \pm 442$ & $3298 \pm 465$ & $+2.3 \pm 5.0 \%$ \\
\hline $\mathrm{PCE}_{\text {low }}$ & $3076 \pm 441$ & $3209 \pm 446$ & $+4.4 \pm 2.5 \%$ **** & $3171 \pm 407$ & $3287 \pm 405$ & $+3.7 \pm 2.7 \% *$ \\
\hline \multicolumn{7}{|c|}{$\dot{V}_{\mathrm{E}}\left(1 \cdot \min ^{-1}\right)$} \\
\hline PCE $E_{\text {high }}$ & $75.3 \pm 11.1$ & $80.5 \pm 11.8$ & $+7.1 \pm 6.5 \% * *$ & $81.1 \pm 11.1$ & $84.1 \pm 13.6$ & $+3.5 \pm 5.4 \% *$ \\
\hline $\mathrm{PCE}_{\text {low }}$ & $77.5 \pm 110$ & $81.8 \pm 12.6$ & $+5.4 \pm 4.4 \% * *$ & $80.0 \pm 11.8$ & $85.6 \pm 13.9$ & $+6.9 \pm 5.1 \%$ *** \\
\hline
\end{tabular}

Measurement of $\dot{V} \mathrm{O}_{2}$ at $100 \mathrm{rpm}$ was conducted at the first $\left(100 \mathrm{rpm}_{\text {start }}\right)$ and at the last $\left(100 \mathrm{rpm}_{\text {end }}\right)$ cadence step during determination of EOC. Whole cycling session was performed twice, once at high fixed cadence ( $\mathrm{PCE}_{\text {high }}$ ) and another at low fixed cadence (PCE $\left.\mathrm{P}_{\text {low }}\right)$ during prolonged cycling exercise

* Statistically significant difference to $100 \mathrm{rpm}_{\text {start }} * P<0.05$, ** $P<0.01, * * * P<0.001$

Fig. 3 Respiratory exchange ratio during determination of energetically optimal cadence before (white diamonds) and after (black diamonds) prolonged cycling exercise (PCE), which was performed either at high $(>95 \mathrm{rpm}, \mathbf{a})$ or low cadence $(<55 \mathrm{rpm}, \mathbf{b})$. Data are presented as mean and standard deviation $(n=14)$. There was a statistically significant reduction in the respiratory exchange ratio after PCE at high $(P<0.01)$ as well as at low cadence $(P<0.001)$

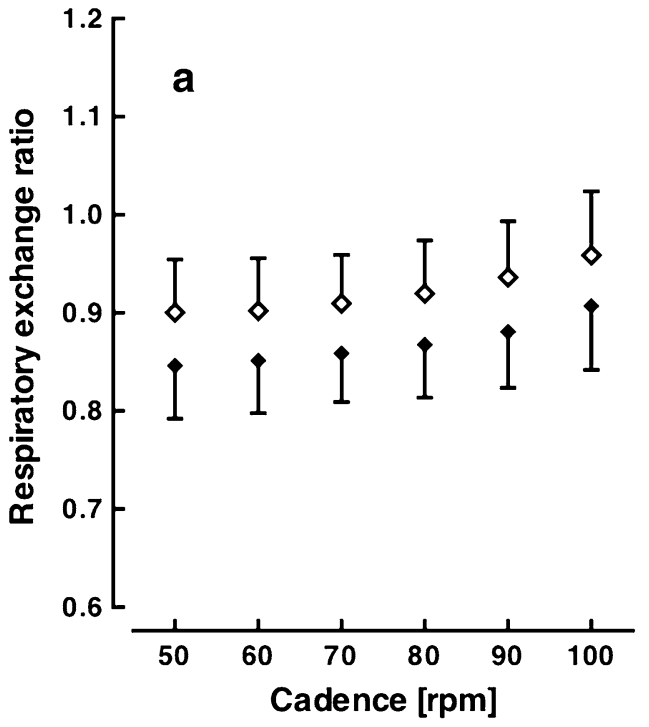

b

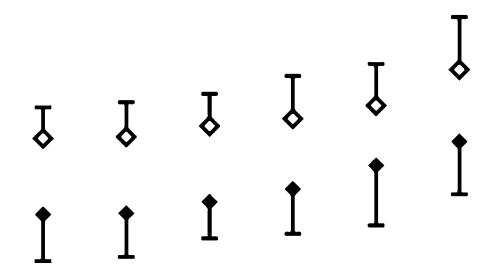

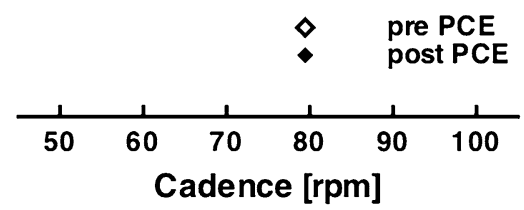




\section{Cardiorespiratory data}

No change in minute ventilation (Fig. 4) was found after the prolonged cycling exercise. The same was true for breathing frequency and tidal volume (data not shown). Heart rate (Fig. 4) increased significantly after the prolonged cycling exercise $(P<0.001)$.

\section{Perceived exertion}

Perceived exertion (Fig. 5) rated significantly higher after the prolonged cycling exercise $(P<0.01)$.

\section{Discussion}

We found a significant reduction in EOC after prolonged cycling $(P<0.01)$. Of 14 subjects, 8 demonstrated a decrease in EOC in both sessions pedalling at a high and a low cadence during the prolonged cycling exercise. The remaining six subjects showed a reduction in EOC at least once, either at a high or at a low cadence. In summary, a reduction in EOC was detected independent of the cadence chosen during the prolonged cycling exercise.

The determination of EOC prior to prolonged cycling exercise gave similar results (58.9 and $59.2 \mathrm{rpm}$ ), although performed on separate days. Prolonged cycling exercise at low cadences induced a more distinctive reduction in EOC $(-21.5 \mathrm{rpm})$ compared to prolonged cycling at high cadences $(-11.3 \mathrm{rpm})$. This difference might be explained by the higher strength required during the low cadence, fatiguing more type II muscle fibres than with the higher cadence as shown by Ahlquist et al. (1992). Whilst external resistance per cycle is lower during cycling exercise at high cadences (Patterson and Moreno 1990), internal work is increased (Francescato et al. 1995). The latter may most
Fig. 4 Minute ventilation (a) and heart rate (b) during determination of energetically optimal cadence before (white diamonds) and after (black diamonds) prolonged cycling exercise (PCE), which was performed either at high ( $>95 \mathrm{rpm}, \mathbf{a})$ or low cadence $(<55 \mathrm{rpm}, \mathbf{b})$. Data are presented as mean and standard deviation $(n=14)$. There was no statistically significant change in minute ventilation after PCE at high $(P=0.981)$ as well as at low cadence $(P=0.890)$. Heart rate was increased after PCE at high $(P<0.01)$ as well as at low cadence $(P<0.001)$
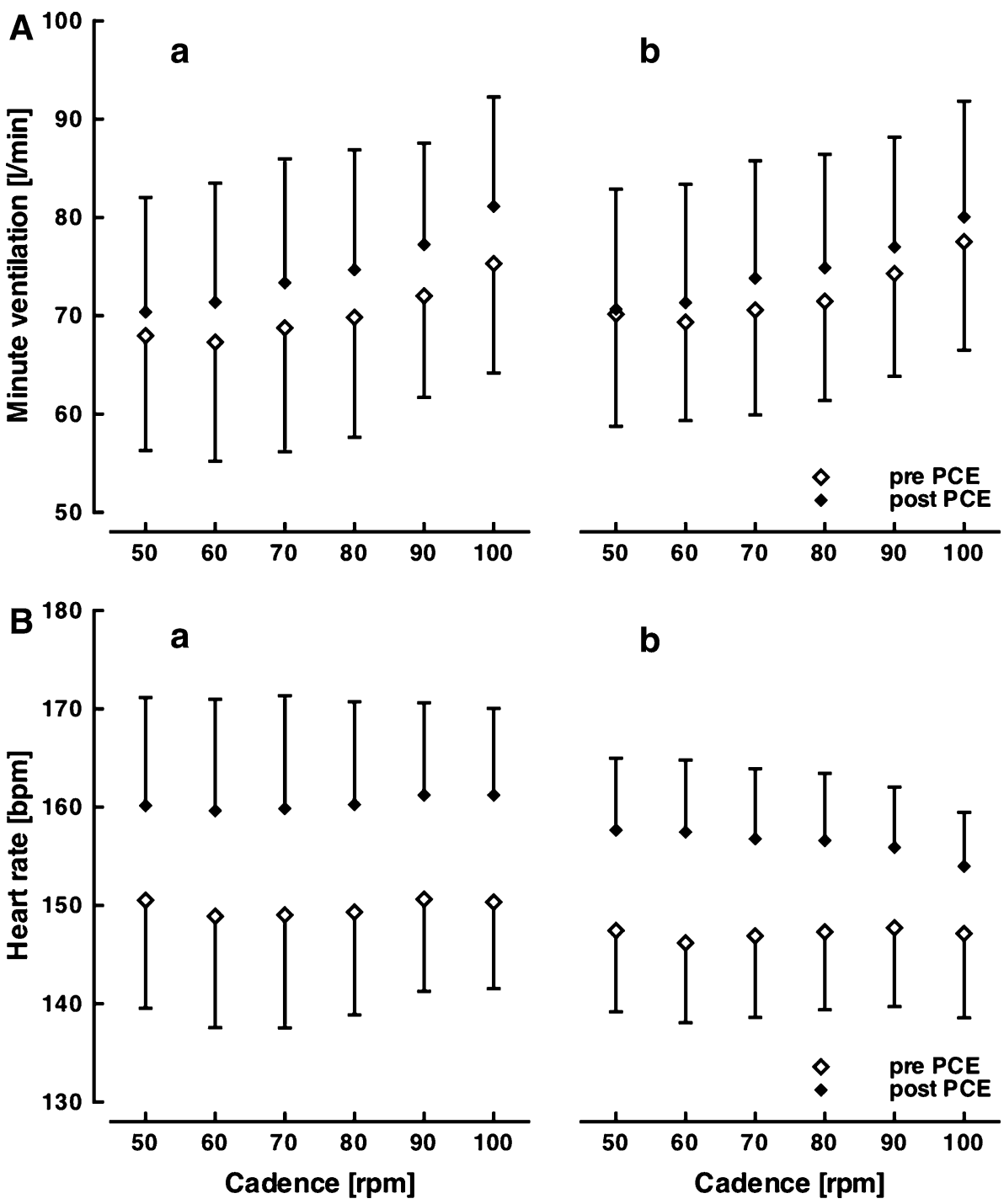

b
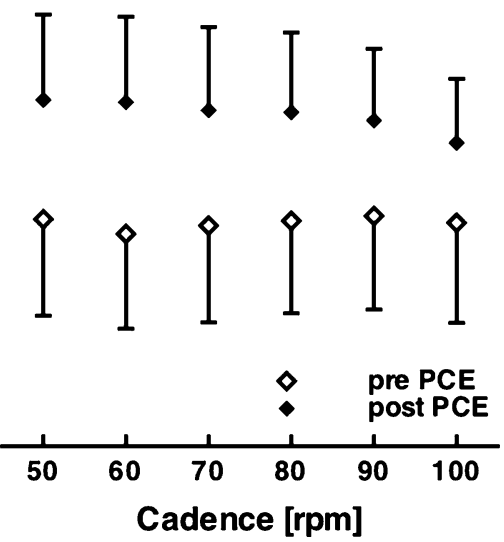
Fig. 5 Rate of perceived exertion at the given power output corresponding to $65 \%$ $\dot{V} \mathrm{O}_{2 \max }$ during determination of energetically optimal cadence before (white diamonds) and after (black diamonds) prolonged cycling exercise (PCE), which was performed either at high $(>95 \mathrm{rpm}, \mathbf{a})$ or low cadence $(<55 \mathrm{rpm}, \mathbf{b})$. Data are presented as mean and standard deviation $(n=14)$. Perceived exertion was rated higher after PCE at high $(P<0.001)$ as well as at low cadence $(P<0.01)$

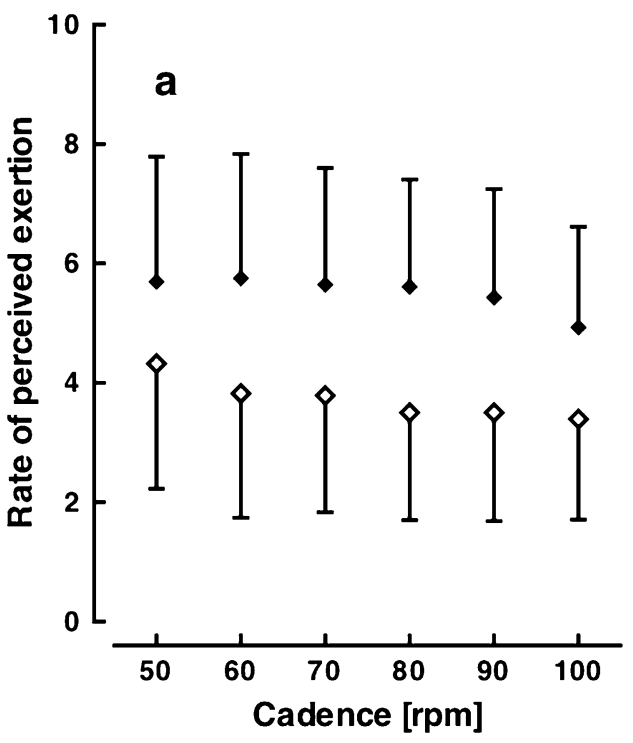

b
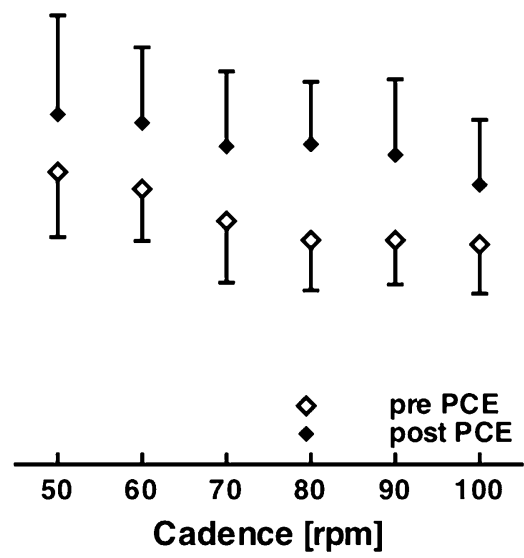

likely put a higher load on the cardiorespiratory system, which could explain the more distinctive increase in minute ventilation and heart rate after cycling at high cadence (Fig. 4). Therefore, the choice of cadence during prolonged cycling exercise reduces EOC to a different extent for different reasons.

Argentin et al. (2006) and Vercruyssen et al. (2001) applied a prolonged cycling protocol with freely chosen cadence. They did not detect a decrease in EOC. Both of them used a comparable intensity to us $\left(65 \% \dot{V} \mathrm{O}_{2 \max }\right.$ or $75 \%$ of maximal heart rate) and prolonged cycling lasted approximately the same duration (77 or $60 \mathrm{~min}$ ). Therefore, the physical demand on subjects can be expected to be similar. We suspect that differences in the determination of EOC led to these different outcomes. Argentin et al. (2006) as well as Vercruyssen et al. (2001) set cadences in a randomised order during determination of EOC. We assume that a randomised order is less sensitive to detect qualitative changes in EOC. Randomisation includes phases where cadence has to be increased. This induces additional work by accelerating lower limbs. Thus, oxygen uptake is affected and time to reach respiratory steady state is probably prolonged. This may lead to uncertainties in detecting EOC, as we have found in preliminary tests. Additionally, as the determination of EOC requires quite a long time to acquire respiratory data at different cadences, a possible effect of the determination on EOC itself cannot be excluded. Therefore, we re-evaluated oxygen consumption at $100 \mathrm{rpm}$ at the end of the determination.

Oxygen consumption was slightly increased at the end of the determination of EOC. It has been shown that endurance exercise for 20-60 min increases oxygen consumption towards the end (e.g. Hagberg et al. 1978; Passfield and Doust 2000). This phenomenon can be attributed for the most part to an increased activity of exercising muscles (Gonzalez-Alonso et al. 1998) and to a lesser extent to increased minute ventilation (Aaron et al. 1992). However, the oxygen upward shift was similar for each determination of EOC (Table 1). Hence, we can probably exclude a significant effect of the EOC protocol on the determination of EOC, especially taking also into consideration that a protocol was applied with a linear decrease in cadence avoiding any additional acceleration of the lower limbs.

Although Argentin et al. (2006) and Vercruyssen et al. (2001) did not find a decrease in EOC, they showed a significant decrease of the freely chosen cadence at the end of prolonged cycling exercise (87-58 rpm, $P<0.01$, or 90-83 rpm). This observation supports our assumption that freely chosen cadence relates to the pattern of activated muscle fibres.

We suggest that the reduction in EOC or of the freely chosen cadence might be induced by a modulation of the pattern of activated muscle fibre types after prolonged cycling exercise. To confirm this speculation, one needs invasive methods, such as the microdissection method, to detect specifically which muscle fibres have been activated (Altenburg et al. 2007). Using surface EMG, Sarre and Lepers (2005) reported an increase in neuromuscular activity throughout a prolonged cycling exercise $(1 \mathrm{~h}$ at $\left.65 \% \dot{V} \mathrm{O}_{2 \max }\right)$. Nevertheless, it is not possible to uniquely identify the activity of individual motor units using the surface EMG (Farina et al. 2004). Recently, Wakeling (2009) presented techniques to detect whether variations occur in the populations of active motor units within a muscle. However, the microdissection method as well as the EMG detects only the activity of the locally investigated muscle parts. Therefore, even with these elaborate 
methods one does not know how relevant the result will be for the entire movement of the lower limb.

If the modulation of the fibre type activation pattern were the only factor affecting whole body measurements and therefore EOC, a conclusion could be drawn from the change in EOC about alterations in muscle fibres activation patterns according to the generalised Hill relationships (Fig. 3 in Kohler and Boutellier 2005). For example, a reduction in EOC of about $5 \mathrm{rpm}$ is based on a divergence of muscle fibre types contributing to muscular work by 6$10 \%$ points. That means that type I muscle fibres contribute to a higher extent to muscular work ( +3 to $+5 \%$ points), whilst the contribution of type II muscle fibres is reduced by the same amount ( -3 to $-5 \%$ points).

The statistically significant decrease in RER (Fig. 3), which indicates an increased fat oxidation after prolonged cycling exercise, supports our interpretation that more type I muscle fibres contribute to muscular work with fatiguing cycling. Energy supply in type I muscle fibres is mainly met by aerobic metabolism compared to type II muscle fibres with a reduced capacity in aerobic energy provision (Bottinelli and Reggiani 2000). Additionally, it is known that oxygen turnover in type I muscle fibres is more efficient than in type II muscle fibres. Therefore, an increase in efficiency is expected after prolonged cycling. However, this was not confirmed by whole body gas exchange though Poole et al. (1992) showed that pulmonary gas exchange correlates highly with leg gas exchange. EE did not change significantly and was even slightly increased. Consequently, gross efficiency showed a slight decrease. Possibly, the efficiency-increasing effect of type I muscle fibres was blunted by the increased oxygen demand of other muscle groups, expressed as increased cardiac and respiratory functions after prolonged cycling exercise (Fig. 4).

Even though these factors reduce gross efficiency, they do no affect EOC since parameters were increased similarly at each cadence (Fig. 4). Furthermore, internal work increases with pedalling frequency, thus enhancing leg gas exchange and EE (Francescato et al. 1995). Widrick et al. (1992) showed that EOC resulting from mechanical efficiency (energy expenditure divided by the sum of external and internal work rate) was consistently higher than that determined from gross efficiency (energy expenditure divided by external work rate). However, limb mass is unchanged by prolonged cycling exercise and therefore the qualitative shift of EOC is not affected by the internal work rate.

In conclusion, the concept of the generalised muscle equations of Hill seems to explain alterations in muscle function and in muscle recruitment during prolonged cycling exercise to maintain external power output. The decreased EOC suggests that more type I muscle fibres contribute to muscular power output after prolonged cycling exercise than before according to the concept of the generalised characteristic muscle equations of Hill. This observation is in accordance with our hypothesis that the activation of muscle fibres changes after prolonged cycling. We think that the determination of EOC is a possible noninvasive method to detect qualitative changes in activated muscle fibres of exercising muscles. This has to be further evaluated and related to results of established methods, such as microdissection methods or EMG.

Acknowledgments We thank Dr. Ruth Briggs for commenting and revising the manuscript. The experiments comply with the current laws of Switzerland.

Conflict of interest statement The authors declare that they have no conflict of interest.

\section{References}

Aaron EA, Seow KC, Johnson JA, Dempsey BD (1992) Oxygen cost of exercise hyperpnea: implications for performance. J Appl Physiol 72:1818-1825

Ahlquist LE, Bassett DR, Sufit R, Nagle DP, Thomas FJ (1992) The effect of pedaling frequency on glycogen depletion rates in type I and type II quadriceps muscle fibers during submaximal cycling exercise. Eur J Appl Physiol 65:360-364

Altenburg TM, Degens H, van Mechelen W, Sargeant A, de Haan AJ (2007) Recruitment of single muscle fibers during submaximal cycling exercise. J Appl Physiol 103:1752-1756

Argentin S, Hausswirth C, Bernard T, Bieuzen F, Leveque JM, Couturier A, Lepers R (2006) Relation between preferred and optimal cadences during two hours of cycling in triathletes. Br J Sports Med 40:293-298

Borg G (1982) Ratings of perceived exertion and heart rates during short-term cycle exercise and their use in a new cycling strength test. Int J Sports Med 3:153-158

Bottinelli R, Reggiani C (2000) Human skeletal muscle fibres: molecular and functional diversity. Prog Biophys Mol Biol $73: 195-262$

Brisswalter J, Hausswirth C, Smith D, Vercruyssen F, Vallier JM (2000) Energetically optimal cadence vs. freely-chosen cadence during cycling: effect of exercise duration. Int $\mathrm{J}$ Sports Med 21:60-64

Chavarren J, Calbet JAL (1999) Cycling efficiency and pedaling frequency in road cyclists. Eur J Appl Physiol 80:555-563

Coast JR, Welch HG (1985) Linear increase in optimal pedal rate with increased power output in cycle ergometry. Eur J Appl Physiol 53:339-342

Di Prampero PE (2000) Cycling on earth, in space, on the moon. Eur J Appl Physiol 82:345-360

Farina D, Macaluso A, Ferguson RA, de Vito G (2004) Effect of power, pedal rate, and force on average muscle fiber conduction velocity during cycling. J Appl Physiol 97:2035-2041

Foss O, Hallén J (2004) The most economical cadence increases with increasing workload. Eur J Appl Physiol 92:443-451

Francescato MP, Girardis M, di Prampero PE (1995) Oxygen cost of internal work during cycling. Eur J Appl Physiol 72:51-57

Gonzalez-Alonso J, Calbet JAL, Nielsen B (1998) Muscle blood flow is reduced with dehydration during prolonged exercise in humans. J Physiol 513:895-905 
Hagberg JM, Mullin JP, Nagle FJ (1978) Oxygen consumption during constant-load exercise. J Appl Physiol 45:381-384

Hansen EA, Andersen JL, Nielsen JS, Sjogaard G (2002) Muscle fibre type, efficiency, and mechanical optima affect freely chosen pedal rate during cycling. Acta Physiol Scand 176:185-194

Henneman E, Somjen G, Carpenter DO (1965) Functional significance of cell size in spinal motoneurons. J Neurophysiol 28:560 580

Hill AV (1938) The heat of shortening and the dynamic constants of muscle. Proc Royal Soc Lond 126:136-195

Hill AV (1964) The effect of load on the heat of shortening of muscle. Proc Royal Soc Lond 159:297-318

Kohler G, Boutellier U (2004) Glycogen reduction in non exercising muscle depends on blood lactate concentration. Eur J Appl Physiol 92:548-554

Kohler G, Boutellier U (2005) The generalized force-velocity relationship explains why the preferred pedaling rate of cyclists exceeds the most efficient one. Eur J Appl Physiol 94:188-195

Martin JC, Brown NA, Anderson FC, Spirduso WW (2000) A governing relationship for repetitive muscular contraction. J Biomech 33:969-974

Passfield L, Doust JH (2000) Changes in cycling efficiency and performance after endurance exercise. Med Sci Sports Exerc 32:1935-1941
Patterson RP, Moreno MI (1990) Bicycle pedalling forces as a function of pedalling rate and power output. Med Sci Sports Exerc 22:512-516

Poole DC, Gaesser GA, Hogan MC, Knight DR, Wagner PD (1992) Pulmonary and leg $\dot{V} \mathrm{O} 2 \max$ during submaximal exercise: implications for muscular efficiency. J Appl Physiol 72:805-810

Sarre G, Lepers R (2005) Neuromuscular function during prolonged pedalling exercise at different cadences. Acta Physiol Scand $185: 321-328$

Vercruyssen F, Hausswirth C, Smith D, Brisswalter J (2001) Effect of exercise duration on optimal pedaling rate choice in triathletes. Can J Appl Physiol 26:44-54

Wakeling JM (2009) Patterns of motor recruitment can be determined using surface EMG. J Electromyogr Kinesiol 19:199-207

Widrick JJ, Freedson PS, Hamill J (1992) Effect of internal work on the calculation of optimal pedaling rates. Med Sci Sports Exerc $24: 376-382$

Zuntz N (1901) Ueber die Bedeutung der verschiedenen Nährstoffe als Erzeuger der Muskelkraft. Pflügers Arch Physiol 83:557 